\title{
Effects of Ionic Liquid and Biomass Sources on Carbon Nanotube Physical and Electrochemical Properties
}

\author{
Kudzai Mugadza ${ }^{1,2}$, Annegret Stark ${ }^{2}$, Patrick G. Ndungu ${ }^{3}(\mathbb{D})$ and Vincent O. Nyamori ${ }^{1, *(D)}$ \\ 1 School of Chemistry and Physics, University of KwaZulu-Natal, Westville Campus, Private Bag X54001, \\ Durban 4000, South Africa; mugadzakudzie@gmail.com \\ 2 SMRI/NRF SARChI Research Chair in Sugarcane Biorefining, School of Engineering, University of \\ KwaZulu-Natal, Durban 4041, South Africa; starka@ukzn.ac.za \\ 3 Energy, Sensors and Multifunctional Nanomaterials Research Group, Department of Chemical Sciences, \\ University of Johannesburg, Doornfontein, Johannesburg 2028, South Africa; pndungu@uj.ac.za \\ * Correspondence: nyamori@ukzn.ac.za
}

Citation: Mugadza, K.; Stark, A.;

Ndungu, P.G.; Nyamori, V.O. Effects of Ionic Liquid and Biomass Sources on Carbon Nanotube Physical and Electrochemical Properties. Sustainability 2021, 13, 2977. https://doi.org/10.3390/su13052977

Academic Editor: Asterios Bakolas

Received: 8 February 2021

Accepted: 2 March 2021

Published: 9 March 2021

Publisher's Note: MDPI stays neutral with regard to jurisdictional claims in published maps and institutional affiliations.

Copyright: (c) 2021 by the authors. Licensee MDPI, Basel, Switzerland. This article is an open access article distributed under the terms and conditions of the Creative Commons Attribution (CC BY) license (https:// creativecommons.org/licenses/by/ $4.0 /)$.

\begin{abstract}
The ongoing research toward meeting global energy demands requires novel materials from abundant renewable resources. This work involves an investigation on nitrogen-doped carbon nanotubes (N-CNTs) synthesized from relatively low-cost and readily available biomass as carbon precursors and their use as electrodes for supercapacitors. The influence of the ionic liquid 1-butyl-3methylimidazolium chloride, or its combination with either sugarcane bagasse or cellulose (IL-CNTs, ILBag-CNTs, and ILCel-CNTs, respectively), in the synthesis of N-CNTs and the resultant effect on their physical and electrochemical properties was studied. Systematic characterizations of the $\mathrm{N}-\mathrm{CNTs}$ employing transmission electron microscopy (TEM), thermogravimetric analysis, X-ray photoelectron spectroscopy (XPS), elemental analysis, nitrogen sorption analysis, cyclic voltammetry, and electrochemical impedance spectroscopy were performed. TEM data analysis showed that the mean outer diameters decreased, in the order of IL-CNTs > ILBag-CNTs > ILCel-CNTs. The N-CNTs possess only pyridinic and pyrrolic nitrogen-doping moieties. The pyridinic nitrogen-doping content is lowest in IL-CNTs and highest in ILCel-CNTs. The N-CNTs are mesoporous with surface areas in the range of 21-52 $\mathrm{m}^{2} \mathrm{~g}^{-1}$. The ILCel-CNTs had the highest specific capacitance of $30 \mathrm{~F} \mathrm{~g}^{-1}$, while IL-CNTs has the least, $10 \mathrm{~F} \mathrm{~g}^{-1}$. The source of biomass is beneficial for tuning physicochemical properties such as the size and surface areas of N-CNTs, the pyridinic nitrogen-doping content, and ultimately capacitance, leading to materials with excellent properties for electrochemical applications.
\end{abstract}

Keywords: carbon nanotubes; biomass; cellulose; sugarcane bagasse; capacitance

\section{Introduction}

Across the globe, the quest for sustainable energy supply has seen societies moving from the extensive usage of petroleum to renewable sources of energy, with active research still ongoing [1]. Mainly, the energy from the sun, which is in abundance, is being highly considered, amongst other alternative reliable sources of energy. The challenge is to effectively harness and store solar energy [2]. Therefore, firstly, photovoltaics are successfully implemented in the conversion of energy from the sun directly into electricity [3]. Secondly, the power converted from the sun is amassed using energy storage devices such as batteries and supercapacitors.

What distinguishes supercapacitors from batteries is their integral constituents and the mechanisms associated with their charge and discharge processes [4]. Supercapacitors possess higher energy-storage and power-delivery capability than batteries [5]. Thus, they are suitable for use in devices where there is a great power demand at relatively short periods. In addition, supercapacitors are associated with a longer life cycle, fast charge and discharge rates coupled with low internal resistance [5]. Hence, they are suitable for applications that require storing high current transient electrical surges to improve the 
efficiency and energy of a system. Supercapacitors are currently gaining popularity in diverse applications, including portable electronic devices, hybrid electric vehicles, and commercial electric utilities [6]. Since there has been an increase in energy demand and reliability, supercapacitors are continuously involved in modification with new electrode materials. These materials allow for operation over a wider potential window with a rapid current response to voltage reversal, which offers high specific capacitances, thus enhancing their storage abilities $[7,8]$.

Mesoporous carbon nanostructured materials (CNMs) are prominent electrode materials for supercapacitors, and this is due to their low cost, exceptional cycle stability, and wide voltage operating windows [9]. Research on the use of biomass-derived CNMs as an electrode material for supercapacitors exists $[7,8]$. However, to the best of our knowledge, this is the first report on the effect of the precursor composition (IL or IL + biomass) on the properties and electrochemical performance of the resulting carbon nanotubes (CNTs).

The unique one-dimensional geometry of CNTs and the associated physicochemical properties such as mechanical strength and excellent electrical properties afford CNTs to be suitable for charge storage and numerous other applications [10]. For achieving a high specific capacitance, a characteristic carbon material should possess a high electroactive surface area and suitable pore sizes that match the size of electrolyte ions [10].

Material engineering by the inclusion of a heteroatom during doping of the carbon material should provide favorable surface characteristics that promote ion intercalation. Of interest is the introduction of heteroatoms such as sulfur, boron, or nitrogen in the graphitic carbon network [11]. N-enriched carbon structures can establish a high capacitance, and this is due to the Faradaic redox reactions, i.e., pseudo-capacitance effects, provided by the nitrogen functional groups [11,12]. Additionally, improved electron donor capability and enhanced surface wetting by the electrolyte can increase the charge storage [12].

Our current work focuses on studying the electrochemical performance of CNTs synthesized using renewable raw materials, namely sugarcane bagasse or cellulose, in combination with ferrocene and an ionic liquid, namely, 1-butyl-3-methylimidazolium chloride $\left(\left[\mathrm{C}_{4} \mathrm{MIM}\right] \mathrm{Cl}\right)$. The primary objectives were to perform capacitance measurements of the N-CNTs and interpret the results with respect to the nitrogen-doping effect, surface area, and tube diameter, amongst others, emanating from the impact of sources. This work contributes advantageously to material beneficiation from available, renewable, and abundant waste biomass for the fabrication of electronic devices such as supercapacitors.

\section{Materials and Methods}

All materials and methods employed are stated, and experimental details are provided in subsequent sections under sample preparation, characterization, and electrochemical measurements.

\subsection{Preparation of the Samples}

$\mathrm{H}_{2} \mathrm{SO}_{4}(98 \%)$ was obtained from Merck, South Africa, $\mathrm{HNO}_{3}(70 \%)$ was obtained from Associated Chemical Enterprises, South Africa. Ferrocene, $98 \%$ (AR), and cellulose Avicel ${ }^{\circledR}$, PH 101, $50 \mu \mathrm{m}$ were purchased from Sigma Aldrich, Germany. Sugarcane bagasse was obtained frozen from the Sugar Milling Research Institute (SMRI). Sugarcane bagasse was shredded to small pieces, sieved, and dried at $100{ }^{\circ} \mathrm{C}$ for $10 \mathrm{~h}$ (weight constant). After drying, the bagasse was ground with mortar and pestle to fine particles.

The ionic liquid (IL), 1-butyl-3-methylimidazolium chloride $\left(\left[\mathrm{C}_{4} \mathrm{MIM}\right] \mathrm{Cl}\right)$, was prepared as previously described [13].

The synthesis of CNTs was conducted with either sugarcane bagasse (Bag) or cellulose (Cel) with ferrocene and $\left[\mathrm{C}_{4} \mathrm{MIM}\right] \mathrm{Cl}(\mathrm{IL})$, following the previously reported procedure (Scheme 1) [13]. 


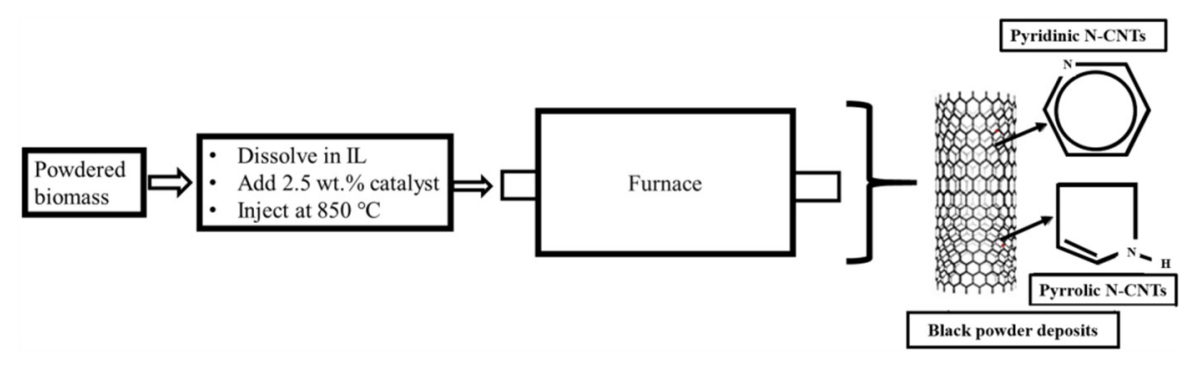

Scheme 1. Schematic presentation of the synthesis of nitrogen-doped carbon nanotubes (N-CNTs).

In short, ferrocene $(0.25 \mathrm{~g})$ and either IL-Bag or IL-Cel solution (containing $10 \%$ of either biomass; $9.75 \mathrm{~g}$ ) were mixed (to make $2.5 \mathrm{wt} \%$ of catalyst) in an ultra-sound sonicator (Digital ultrasonic heater UD150SH-6L) for approximately $20 \mathrm{~min}$. Then, the mixture was transferred into a $10 \mathrm{~mL}$ plastic syringe and set for injection using a purpose-built injection system. The injection system had the requisite gas fittings and was designed to deliver the mixture directly into the centre of a quartz tube located within a horizontally aligned tube furnace.

A horizontally aligned tube furnace, fitted with a main zone temperature controller, was set to the desired temperature using a ramp rate of $10^{\circ} \mathrm{C} / \mathrm{min}$. The carrier gas, $10 \%$ $\mathrm{H}_{2}$ balanced in $\mathrm{Ar}$, was switched on at $600^{\circ} \mathrm{C}$, and a flow rate of $100 \mathrm{~mL} / \mathrm{min}$ was applied. When the respective desired temperature was reached, injection of the sonicated mixture into the chemical vapour deposition (CVD) system at a set injection rate of $0.8 \mathrm{~mL} / \mathrm{min}$ followed. The reaction was set for $30 \mathrm{~min}$, and after that, the furnace was allowed to cool down to room temperature. All the samples collected were purified. The purification process involved calcining all samples using a muffle furnace at $300{ }^{\circ} \mathrm{C}$ in air for $3 \mathrm{~h}$, followed by ultrasonication in $\mathrm{H}_{2} \mathrm{SO}_{4} / \mathrm{HNO}_{3}$ (3:1 by volume) for 40 min using an ultrasonic heater. Thereafter, the mixture was refluxed at $100{ }^{\circ} \mathrm{C}$ for $24 \mathrm{~h}$ at a constant stirring speed of $300 \mathrm{rpm}$. After refluxing, the acid was neutralized with a solution of $\mathrm{NaOH}$, and the mixture was ultrasonicated for $1 \mathrm{~h}$. After filtration and washing with deionized water (until the $\mathrm{pH}$ was 7), the materials were dried at $120^{\circ} \mathrm{C}$ for $3 \mathrm{~h}$. The CNTs synthesized from IL and ferrocene are denoted IL-CNTs, while those from the IL-bagasse and IL-cellulose mixtures are denoted as ILBag-CNTs and ILCel-CNTs, respectively.

\subsection{Characterization}

Characterization of the carbon nanostructures utilized TEM (JEOL JEM 1010) with the aid of Image J software for structural dimension determination. Elemental analysis was performed using an LECO CHNS-932 elemental analyzer standardized with acetanilide. X-ray photoelectron spectroscopy (XPS) analysis was performed with a Thermo ESCAlab 250Xi equipped with monochromatic $\mathrm{Al} \mathrm{K} \alpha(1486.7 \mathrm{eV})$ X-rays. An X-ray power of $300 \mathrm{~W}$, with an X-ray spot size of $900 \mu \mathrm{m}$, pass energy (Survey) of $100 \mathrm{eV}$, pass energy (Hiresolution) of $20 \mathrm{eV}$, and pressure of $10^{-8}$ mbar were used. The thermogravimetric analysis utilized the TA instruments Q seriesTM Thermal Analyzer DSC/TGA (Q600) with a TA instruments universal analysis 2000 software for data acquisition. The samples were run in oxygen at a $100 \mathrm{~mL} / \mathrm{min}$ flow rate and from ambient temperatures to $1000{ }^{\circ} \mathrm{C}$ at $10{ }^{\circ} \mathrm{C} / \mathrm{min}$. Nitrogen sorption studies were performed with a Micromeritics TriStar 3020 V1.03 (V1.03) instrument. Surface area and porosity were calculated with the use of Brunauer-Emmett-Teller (BET) and Barrett-Joyner-Halenda (BJH) methods, respectively.

\subsection{Electrochemical Measurements}

The electrochemical tests were performed using cyclic voltammetry $(\mathrm{CV})$ and electrochemical impedance spectroscopy (EIS) on an electrochemical CHI 660E work station, $\mathrm{CH}$ Instruments, Austin, Texas, USA. All the electrochemical measurements were achieved by the use of a three-electrode system. The counter and reference electrodes were Pt and $\mathrm{Ag} / \mathrm{AgCl}$, respectively. The working electrode was prepared by drop-casting the sample 
mixed with Nafion onto a glassy carbon electrode ( $3 \mathrm{~mm}$ diameter). The deposited mixture, on the electrode, was dried at room temperature. The electrolyte, $\mathrm{H}_{2} \mathrm{SO}_{4}(0.1 \mathrm{M})$, was prepared by diluting $98 \%$ solution with double deionized water. The electrodes used for EIS were likewise prepared.

\section{Results and Discussions}

Effects of the precursor on the physicochemical properties of the synthesized CNTs and the electrochemical performance are reported herein.

\subsection{Morphological Analysis}

The TEM image analysis of the three samples shows hollow tubular structures with some bamboo compartments (Figure 1), which resemble nitrogen-doped CNTs $[14,15]$. The outer diameters (ODs) of the CNTs after purification are 101, 63, and $38 \mathrm{~nm}$ for IL-CNTs, ILBag-CNTs, and ILCel-CNTs, respectively. The differences in the size of CNT diameters were attributed to the presence of oxygen in the biomass containing precursors. Oxygen affects the activation of the catalyst, the decomposition of the carbon precursor, and also acts as an etchant of amorphous carbon [16]. Additionally, the diameters of the purified samples are smaller than those from the crude samples $(172,104$, and $112 \mathrm{~nm}$ for IL-CNTs, ILBag-CNTs, and ILCel-CNTs, respectively). Similarly, the reduction in sizes was attributed to the etching effect of the purification processes.

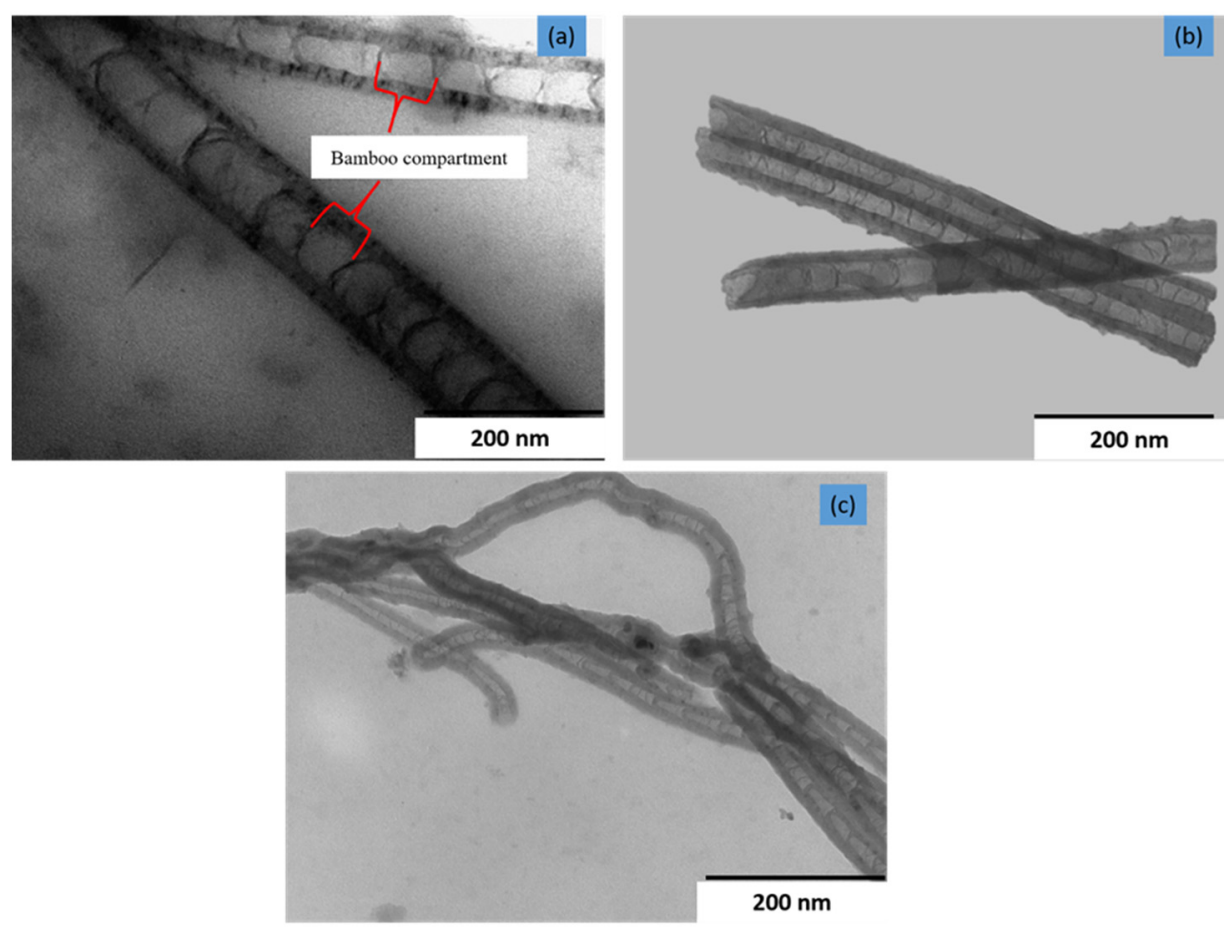

Figure 1. TEM images for the synthesized structures: (a) IL-CNTs, (b) ILBag-CNTs and (c) ILCelCNTs. IL-CNTs, ILBag-CNTs, and ILCel-CNTs: ionic liquid 1-butyl-3-methylimidazolium chloride, or its combination with either sugarcane bagasse or cellulose.

Apart from being smaller, the ILCel-CNTs are notably more bent than the IL-CNTs and ILBag-CNTs (Figure 1). The inward bent structure defects suggest that pentagons were formed because of nitrogen doping [13].

\subsection{Chemical Composition Analysis}

Elemental analysis (EA) was initially used for qualitative analysis and showed the presence of carbon, nitrogen, hydrogen, and oxygen in the samples (Supplementary Information, Figure S1). IL-CNTs have a higher content of doped N, followed by ILBag-CNTs 
and ILCel-CNTs. This concurs with the TEM observations where IL-CNTs were found to have more pronounced bamboo compartments. Further elemental quantitative analysis through XPS shows a trend of atomic nitrogen composition similar to that from EA, i.e., thus confirming a higher percentage of nitrogen in the IL-CNTs sample (Figure 2). The atomic nitrogen content for IL-CNTs, ILBag-CNTs, and ILCel-CNTs are 7.2\%, 7.1\%, and $6.2 \%$, respectively (Figure 2 ).

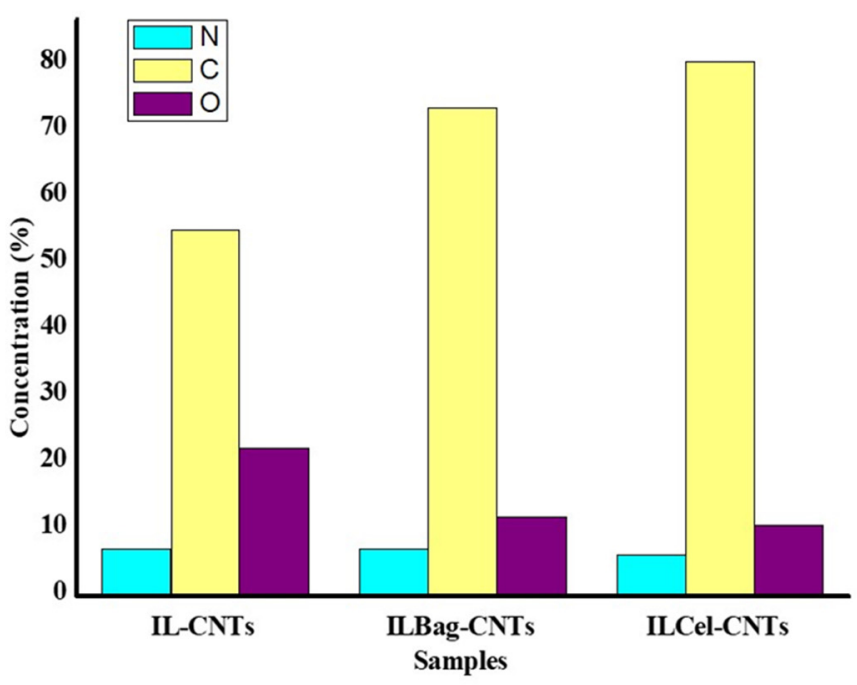

Figure 2. Elemental composition of $\mathrm{C}, \mathrm{N}$, and $\mathrm{O}$ in all samples from XPS.

The N1s peak show a broad peak between 397.7 and $400.4 \mathrm{eV}$ (Figure 3a-c), which was deconvoluted to reveal the presence of two major nitrogen-doped species in the carbon lattices, namely pyridinic and pyrrolic around 397.6-398.5 and 399.6-400.5 eV, respectively [17-20]. These peaks confirm the presence of nitrogen doping in the samples, which concurs with the presence of bent structures and bamboo compartments as observed using TEM (Figure 1).
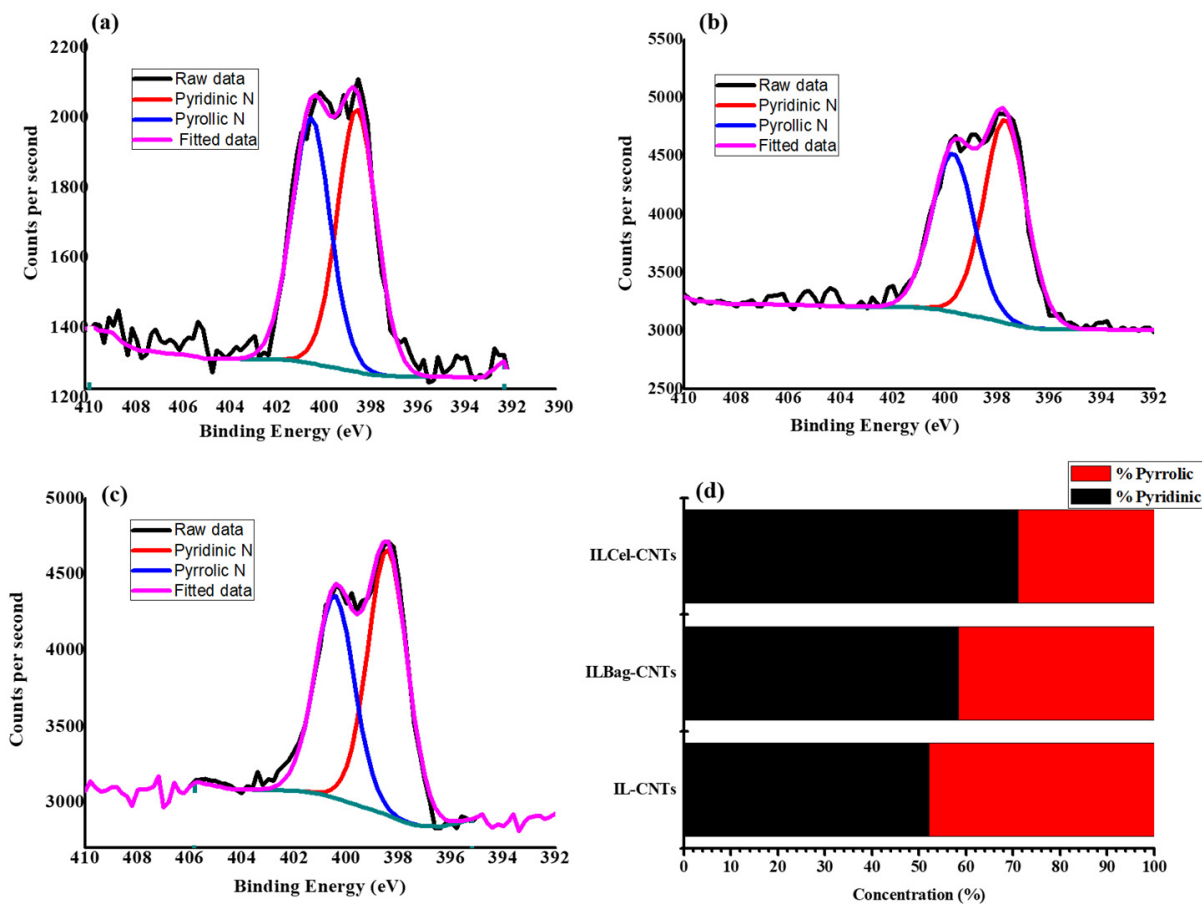

Figure 3. XPS nitrogen species in samples (a) IL-CNTs, (b) ILBag-CNTs, (c) ILCel-CNTs, and (d) composition of these deconvoluted $\mathrm{N}$ species in the materials. 
The deconvoluted N1s species ratio was used to determine the percentage composition of the identified species (Figure 3d). In all samples, the percentage of the pyridinic nitrogen is higher than the pyrrolic nitrogen, i.e., $52 \%, 58 \%$, and 71\% IL-CNTs, ILBag-CNTs, and ILCel-CNTs, respectively. Interestingly, ILCel-CNT, which shows bent structures in the TEM analysis (Figure 1c), has the lowest pyrrolic-nitrogen content, and thus, the inclusion of the unsymmetrical pyrrole ring is not responsible for this phenomenon. Hence, these results suggest that the more pronounced bent structures of ILCel-CNTs are due to the smaller tube sizes.

\subsection{Thermal Analysis}

The thermogravimetric analysis (TGA) profiles of the CNTs show minimal water loss $\left(\approx 2 \mathrm{wt} \%\right.$ ), which is expected to occur below $200{ }^{\circ} \mathrm{C}$ (Figure 4). There is no noticeable decrease in weight in the range of $200-400{ }^{\circ} \mathrm{C}$, corroborating TEM analysis and inferring the absence of amorphous carbon. Significant weight loss occurs from around $500{ }^{\circ} \mathrm{C}$, with a weight loss of $\approx 95 \mathrm{wt} \%$ from both ILCel-CNTs and IL-CNTs, at 630 and $611^{\circ} \mathrm{C}$, respectively, and $\approx 97 \mathrm{wt} \%$ from ILBag-CNTs samples at $618^{\circ} \mathrm{C}$. This was assigned to the decomposition of N-CNTs, which is attributed to the disintegration of a single type of $\mathrm{sp}^{2}$ carbon structures. This is further supported by TEM analysis where no other structures such as spheres, fibers, or amorphous carbons were observed (Figures 1 and 4). In addition, the XPS analysis further supports the findings from the TGA analysis.

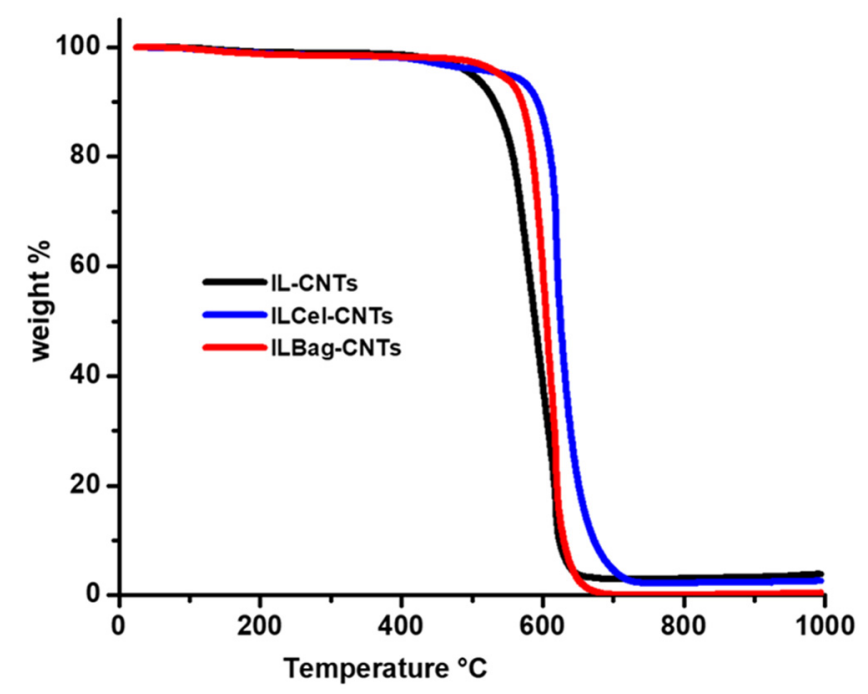

Figure 4. Thermal analysis profiles of IL-CNTs, ILCel-CNTs, and ILBag-CNTs samples.

The thermogram in Figure 4 indicates that the order of material tolerance to heat is IL-CNTs $<$ ILBag-CNTs $<$ ILCel-CNTs, i.e., $\mathrm{T}_{\max }$ DTA of 611,618 , and $630{ }^{\circ} \mathrm{C}$, respectively. These thermal stability trends agree with the amount of doped $\mathrm{N}$ determined by XPS. The thermal decomposition temperatures are close to each other, and this is due to the similar relationship of nitrogen contents, as seen in Figure 2. IL-CNTs show the highest amount of doped $\mathrm{N}$ within the graphitic structure; hence, they have relatively higher compromised $\mathrm{C}-\mathrm{C}$ bond strengths and poorer heat tolerance. Some residual Fe remains in the materials, as evidenced by the incomplete decomposition. The sample containing ILBag-CNTs has almost zero residual Fe content while ILCel-CNTs and IL-CNTs have a residual Fe content of around $3 \mathrm{wt} \%$. This could be due to non-dissolved Fe after the purification process since the concentrated $\mathrm{H}_{2} \mathrm{SO}_{4} / \mathrm{HNO}_{3}$ mixture has the ability to dissolve the metal catalyst, separate the CNTs, and remove carbonaceous impurities.

\subsection{Surface Area and Porosity Analysis}

Nitrogen sorption and desorption data can be used to determine the surface area, pore size distribution, as well as the ratio of micro- to mesopores. Surface area and porosity 
were calculated with the BET and BJH methods, respectively. The samples exhibit type IV sorption-desorption isotherms with an $\mathrm{H} 2$ hysteresis loop observed in the range of $0.45-1.0 \mathrm{P} / \mathrm{P}_{0}$ (Figure 5a).
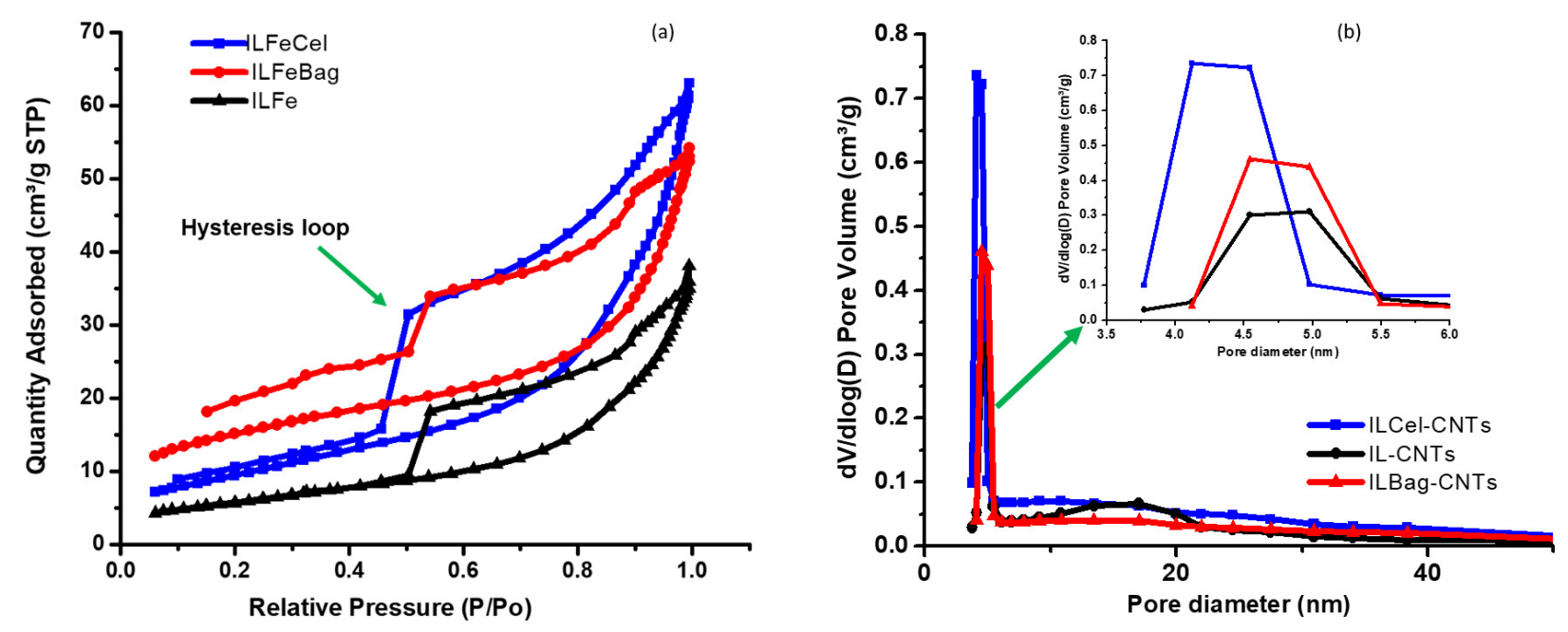

Figure 5. (a) Nitrogen adsorption and desorption isotherms; and (b) pore size distribution.

The $\mathrm{H} 2$ hysteresis loop suggests the presence of pores with narrow mouths (ink-bottle pores), comparatively constant channel-like pores, and pore network-linking effects [21], therefore suggesting the samples are mesoporous materials. The hysteresis loop of ILCelCNTs is slightly broader than the other two, suggesting the presence of more porous structures with possibly greater surface area and pore volume. This observation correlates to the highest BET surface area of $52 \mathrm{~m}^{2} \mathrm{~g}^{-1}$ for ILCel-CNTs, which is followed by ILBagCNTs, $35 \mathrm{~m}^{2} \mathrm{~g}^{-1}$, and IL-CNTs with the lowest surface area of $21 \mathrm{~m}^{2} \mathrm{~g}^{-1}$. These surface areas correspond to the increase in ODs of the CNTs, i.e., 38, 63, and $101 \mathrm{~nm}$, respectively, hence suggesting that the smaller the OD, the greater the surface area. The pore size distribution of samples is displayed in Figure $5 b$, and the insert is for the size region of 3.5-5.5 nm. The order of population frequency of these pores was IL-CNTs < ILBagCNTs < ILCel-CNTs, respectively. The most probable reason for this is the different dimensions and composition of nitrogen-doping types leading to altered structures and textural characteristics. The pore distribution indicates that the CNTs are mostly composed of mesopores $(2-50 \mathrm{~nm})$.

\subsection{Cyclic Voltammetry}

Cyclic voltammetry (CV) is an effective and popular electrochemical technique frequently utilized to study the electrochemical performance of materials. The charge accumulation at electrodes was determined using a scan rate of $10 \mathrm{mV} \mathrm{s}^{-1}$ in a potential range of 0 to $0.8 \mathrm{~V}$. The specific capacitance (Cs) was calculated from the CV curves according to Equation (1) [22]:

$$
C s=\frac{\int I d V}{2 v \Delta V m}
$$

where $\mathrm{Cs}$ is the specific capacitance $\left(\mathrm{F} \mathrm{g}^{-1}\right)$, I is the current response $(\mathrm{A}), \Delta \mathrm{V}$ is the potential window $(\mathrm{V}), v$ is the scan rate $(\mathrm{V} / \mathrm{s})$, and $\mathrm{m}$ is the mass of electrode material $(\mathrm{g})$. The capacitive performance of the CNTs was obtained in $0.1 \mathrm{M} \mathrm{H}_{2} \mathrm{SO}_{4}$ at room temperature. The fabricated electrodes from all the N-CNT materials display some pseudo-capacitance traits and rectangular-shaped CV curves (Figure 6). This means that all the N-CNT materials investigated show an excellent rapid current response to voltage reversal and excellent electrochemical capacitive behavior. A pair of reversible redox peaks at 0.43 and $0.53 \mathrm{~V}$ was attributed to the presence of $\mathrm{N}$ introducing Faradic reactions between nitrogen atoms in the N-CNT matrix and the electrolyte. In acidic medium, the pseudo-Faradaic effects 
resulting from CNTs with pyridinic nitrogen functionality can be characterized according to Equation (2) $[18,23]$ :

$$
\mathrm{C}^{*}=\mathrm{N}+\mathrm{e}^{-}+\mathrm{H}^{+} \leftrightarrow \mathrm{C}^{*} \mathrm{NH}
$$

where $C^{*}$ stands for the carbon network. There were no oxidation and reduction peaks of Fe observed in CV curves, and this observation suggests that the effect of the residual Fe nanoparticles from synthesis is negligible $[24,25]$.

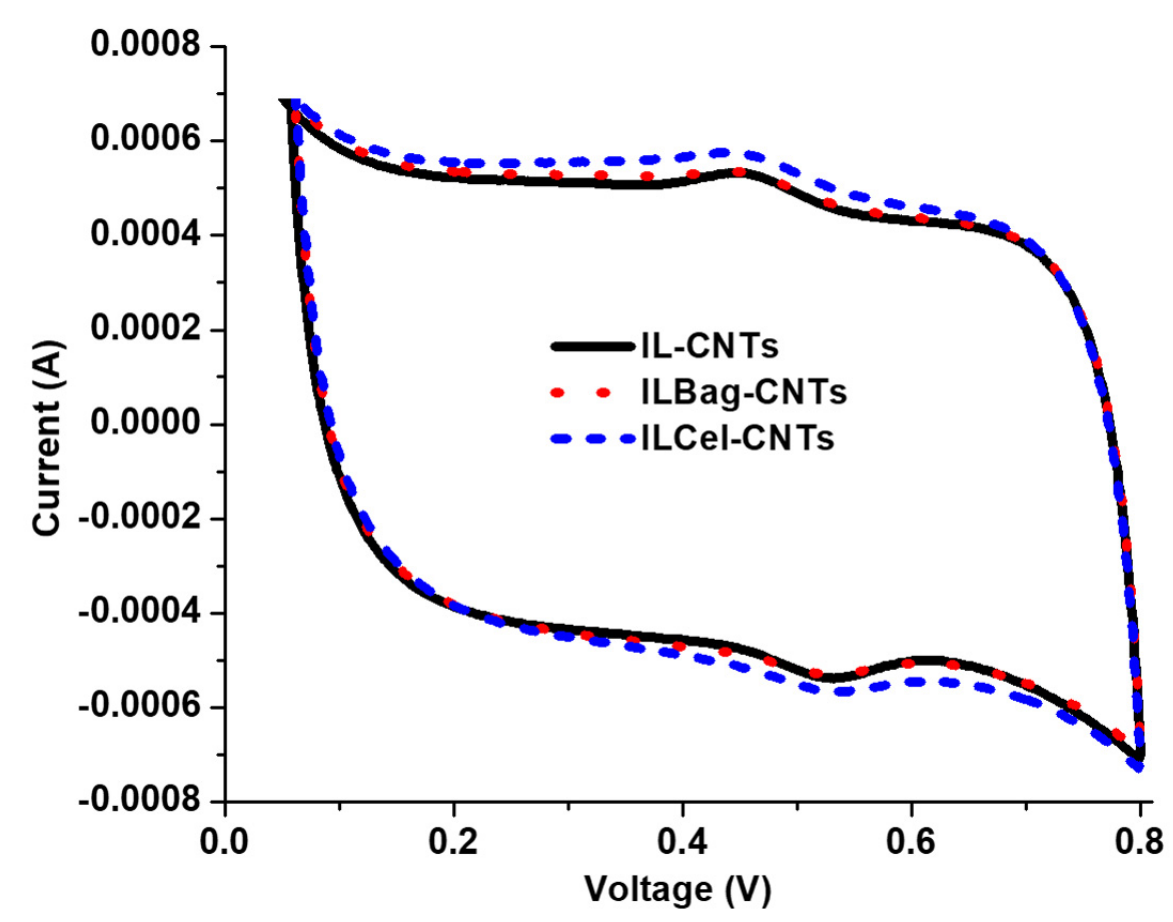

Figure 6. Cyclic voltammetry (CV) curves of all nanostructures at a scan speed of $10 \mathrm{mV} \mathrm{s}^{-1}$.

The existence of nitrogen as a dopant in CNTs results in enhanced capacitance due to the modified electronic properties [26]. However, an increase in pyrrolic nitrogen-doping in $\mathrm{N}-\mathrm{CNTs}$ has been reported to cause severe deterioration regarding both the electrochemical capacitor (EC) quality and the current [27]. However, in the present work, the EC quality did not deteriorate with an increase in pyrrolic nitrogen doping (Figure 6).

Furthermore, introducing nitrogen, an electron-rich atom, in the form of pyridinic moieties in the graphitic carbon network adds a lone pair of electrons into the delocalized $\pi$ system [19]. The function of the lone pair of electrons is to act as a charge carrier, which improves the charge density and, in turn, leads to increased electrical conductivity, therefore resulting in increased capacitance [28]. It is also important to note that the substitution of carbon with nitrogen provides natural carbon materials with other redox reactions for pseudocapacitors [29]. On the other hand, pyrrolic nitrogen is known to induce a high concentration of defects within the graphitic structure since five-membered rings are formed instead of six-membered rings [30], which compromise the associated electrical conductivity of carbon and cause a loss of capacitance.

The synthesized N-CNTs exhibit Cs of 10, 26, and $30 \mathrm{~F} \mathrm{~g}^{-1}$ for IL-CNTs, ILBag-CNTs, and ILCel-CNTs, respectively. The Cs is comparable to the reported values of commercially available doped N-CNTs [31]. The total nitrogen content in the samples is very similar (7.2\%, 7.1\%, and $6.2 \%$ for IL-CNTs, ILBag-CNTs, and ILCel-CNTs), but the amount of pyrrolic N-doping decreases in the series (48\%, 42\%, and 29\% (Figure 3d)) and is hence in line with the literature. The data indicate that the performance of the supercapacitors can be tuned by pyridinic nitrogen-doping composition.

Another reason for the increase in specific capacitance in that series could be the different porosities and specific surface areas [23]. Sufficiently large surface areas must be 
accessible to the electrolyte ions for adsorption and ultimately forming a double layer. In the above series, both the surface area and the average pore volume increase, indicating indirectly that the surface area is available for electrosorption. This contrasts to other reports [32,33], where no relationship between EC quality and performance with BET surface area was found. This work indicates that the physical properties, namely, diameter, porosity, and surface area of N-CNTs, are suitably tailored by introducing cellulose or bagasse as feedstock combined with the ionic liquid.

Cycle stability studies were performed on all three samples at $50 \mathrm{mV} \mathrm{s}^{-1}$ for 50 cycles (Figure 7a).
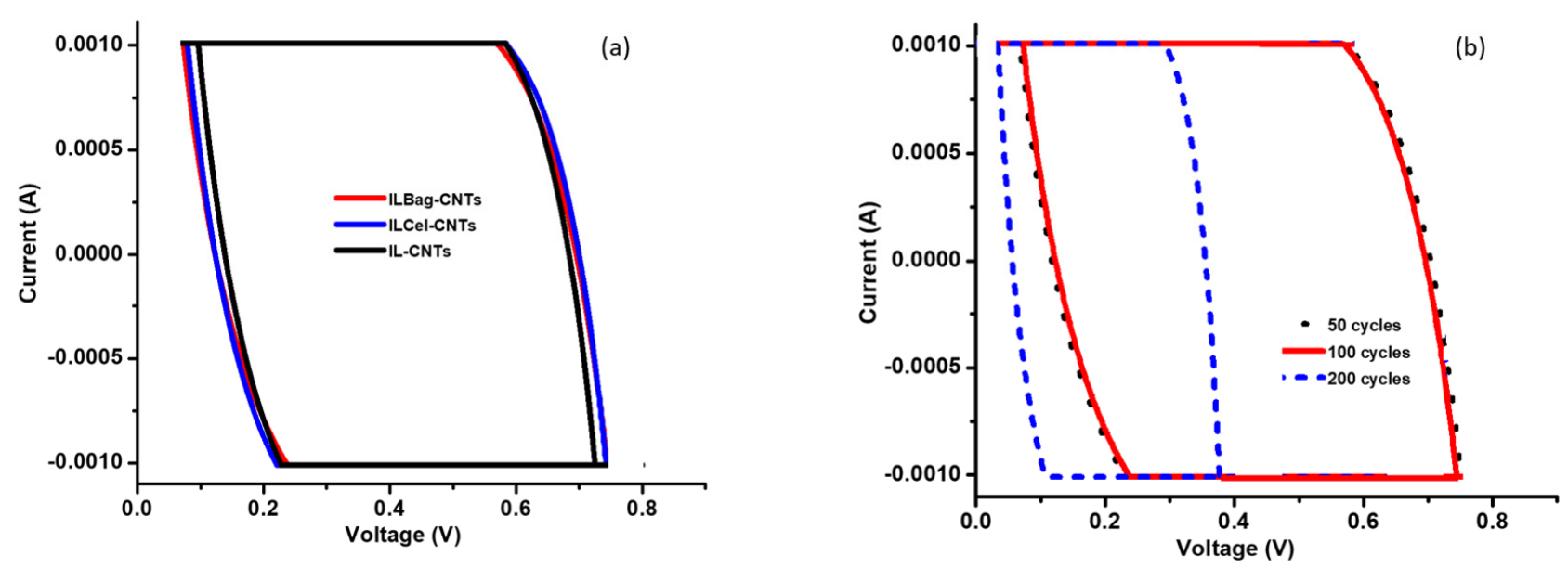

Figure 7. Cycle stability of (a) all samples at $50 \mathrm{mV} \mathrm{s}^{-1}$; (b) ILCel-CNTs at 50, 100, and 200 cycles at $50 \mathrm{mV} \mathrm{s}^{-1}$.

It is observed that all samples are stable, with ILCel-CNTs being slightly more stable based on the voltage window. Therefore, the most stable sample, ILCel-CNTs, which also features the best capacitive performance, was further studied for long-term cycle stability studies, i.e., 50, 100, and 200 cycles, as shown in Figure 7b. The voltammogram maintained its quasi-rectangular shape at 50 and 100 cycles, at a scan rate of $50 \mathrm{mV} \mathrm{s}^{-1}$, indicating quick and efficient charge transfer and excellent capacitive behavior [34]. However, it deteriorated severely between the 100th and 200th cycles, as shown by the smaller voltage window (Figure $7 b$ ).

\subsection{Electrochemical Impedance Spectroscopy (EIS)}

Nyquist plots display the frequency responses of the samples, which demonstrate the associated impedance properties of the CNTs [35]. The high-frequency region shows negligible charge transfer resistance for all samples (Figure 8). Ideally, in cases where there is charge transfer resistance, the Nyquist plot may show a distorted semicircle in the high-frequency region. However, in this case, the semicircle is absent, indicating that the resistance is negligible, and hence, there is a remarkable charge reversal at voltage change, leading to the rectangular shapes shown in Figure 6 for the $C V$ analysis.

The lower frequency region of the three CNT materials shows a straight line, which is attributed to the low ion diffusion resistance in the electrolyte, suggesting a nearly ideal charge storage device [35]. The slope for ILCel-CNTs is slightly steeper than those for ILBag-CNTs and IL-CNTs, suggesting faster ion diffusion and better EC quality. 


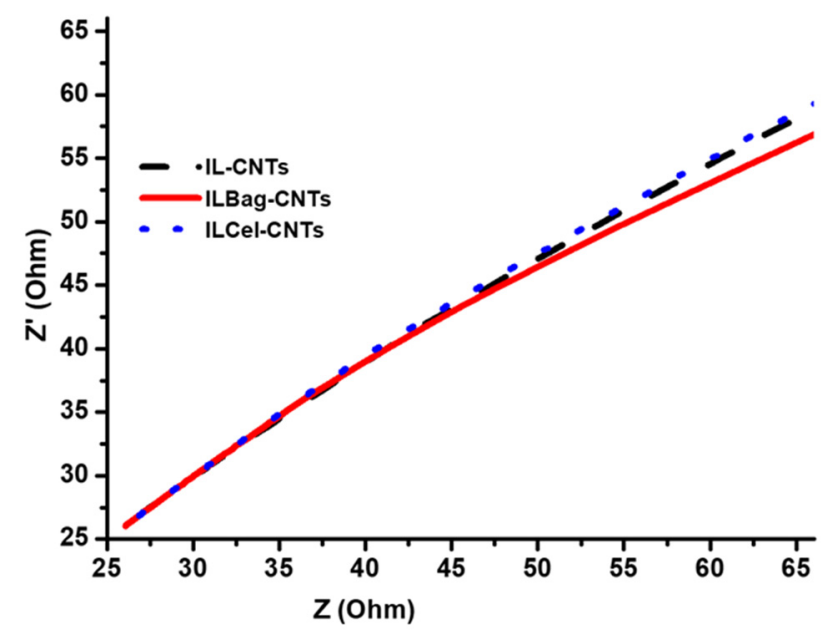

Figure 8. Nyquist plot.

\section{Conclusions}

In conclusion, the results have shown that excellent electrochemical properties are imparted to N-CNTs when these are prepared from 1-butyl-3-methylimidazolium chloride or a mixture of this ionic liquid with either bagasse or cellulose. Hence, the developed protocol allows fabricating electronic devices such as supercapacitors from renewable resources. Depending on the starting material composition, the physical (dimensions, pore size distribution, surface area), chemical (elemental composition, level of N-doping, ratio of pyridinic and pyrollic nitrogen, thermal stability) and electrochemical properties electrochemical capacitor, EC, (EC quality, specific capacitance, impedance) of the material are tailored. In particular, the N-CNTs synthesized from cellulose display excellent performance.

Supplementary Materials: The following are available online at https:/ / www.mdpi.com/2071-105 0/13/5/2977/s1.

Author Contributions: Conceptualization, K.M.; methodology, K.M.; validation, K.M., A.S., P.G.N. and V.O.N.; formal analysis, K.M.; investigation, K.M.; data curation, K.M.; writing—original draft preparation, K.M.; writing-review and editing, A.S., P.G.N., and V.O.N.; supervision, A.S., P.G.N. and V.O.N. All authors have read and agreed to the published version of the manuscript.

Funding: This work is based on the research supported in part by grant numbers 116610 (KM), 103979 (VON), 115465 (AS) by the National Research Foundation of South Africa.

Institutional Review Board Statement: Not applicable.

Informed Consent Statement: Not applicable.

Data Availability Statement: Data is contained within the article or Supplementary Materials.

Acknowledgments: The authors would like to thank the Synthetic and Medicinal Chemistry Research Group (SMCRG) at the University of KwaZulu-Natal for the use of the CHI instruments. Kudzai Mugadza wishes to thank the Sugar Milling Research Institute (SMRI) and the National Research Foundation-The World Academy of Science (NRF-TWAS) for the supply of the feedstock and funding, respectively.

Conflicts of Interest: The authors declare no conflict of interest.

\section{References}

1. Dresselhaus, M.S.; Thomas, I.L. Alternative energy technologies. Nature 2001, 414, 332-337. [CrossRef] [PubMed]

2. Lewis, N.S.; Nocera, D.G. Powering the planet: Chemical challenges in solar energy utilization. Proc. Natl. Acad. Sci. USA 2006, 103, 15729-15735. [CrossRef] [PubMed]

3. Green, M.A.; Bremner, S.P. Energy conversion approaches and materials for high-efficiency photovoltaics. Nat. Mater. 2016, 16, 23-34. [CrossRef] [PubMed] 
4. Dubal, D.P.; Ayyad, O.; Ruiz, V.; Gomez-Romero, P. Hybrid energy storage: The merging of battery and supercapacitor chemistries. Chem. Soc. Rev. 2015, 44, 1777-1790. [CrossRef]

5. Chen, H.; Cong, T.N.; Yang, W.; Tan, C.; Li, Y.; Ding, Y. Progress in electrical energy storage system: A critical review. Prog. Nat. Sci. 2009, 19, 291-312. [CrossRef]

6. Kötz, R.; Carlen, M. Principles and applications of electrochemical capacitors. Electrochim. Acta 2000, 45, 2483-2498. [CrossRef]

7. Wang, H.; Yang, Y.; Guo, L. Nature-inspired electrochemical energy-storage materials and devices. Adv. Energy Mater. 2017, 7, 1601709. [CrossRef]

8. Li, B.; Dai, F.; Xiao, Q.; Yang, L.; Shen, J.; Zhang, C.; Cai, M. Nitrogen-doped activated carbon for a high energy hybrid supercapacitor. Energy Environ. Sci. 2016, 9, 102-106. [CrossRef]

9. Bairi, P.; Shrestha, R.G.; Hill, J.P.; Nishimura, T.; Ariga, K.; Shrestha, L.K. Mesoporous graphitic carbon microtubes derived from fullerene $\mathrm{C} 70$ tubes as a high performance electrode material for advanced supercapacitors. J. Mater. Chem. A 2016, 4, 13899-13906. [CrossRef]

10. Zhang, Q.; Huang, J.-Q.; Qian, W.-Z.; Zhang, Y.-Y.; Wei, F. The road for nanomaterials industry: A review of carbon nanotube production, post-treatment, and bulk applications for composites and energy storage. Small 2013, 9, 1237-1265. [CrossRef]

11. Fic, K.; Frackowiak, E.; Béguin, F. Unusual energy enhancement in carbon-based electrochemical capacitors. J. Mater. Chem. 2012, 22, 24213-24223. [CrossRef]

12. Wickramaratne, N.P.; Xu, J.; Wang, M.; Zhu, L.; Dai, L.; Jaroniec, M. Nitrogen enriched porous carbon spheres: Attractive materials for supercapacitor electrodes and $\mathrm{CO}_{2}$ adsorption. Chem. Mater. 2014, 26, 2820-2828. [CrossRef]

13. Mugadza, K.; Ndungu, P.G.; Stark, A.; Nyamori, V.O. Ionic liquids and cellulose: Innovative feedstock for synthesis of carbon nanostructured material. Mater. Chem. Phys. 2019, 234, 201-209. [CrossRef]

14. Labulo, A.H.; Ngidi, N.P.; Omondi, B.; Nyamori, V.O. Physicochemical properties of nitrogen-doped carbon nanotubes from metallocenes and ferrocenyl imidazolium compounds. J. Organomet. Chem. 2018, 868, 66-75. [CrossRef]

15. Steinmetz, M.; Lima, D.; Machado RR, L.; Sundararaj, U.; Arjmand, M.; da Silva, A.B.; Santos, J.P.; Pessôa, C.A.; Wohnrath, K. Nitrogen-doped carbon nanotubes towards electrochemical sensing: Effect of synthesis temperature. Diam. Relat. Mater. 2020, 110, 108093. [CrossRef]

16. Mori, S.; Suzuki, M. Effect of oxygen and hydrogen addition on the low-temperature synthesis of carbon nanofibers using a low-temperature CO/Ar DC plasma. Diam. Relat. Mater. 2008, 17, 999-1002. [CrossRef]

17. Hassan, F.M.; Chabot, V.; Li, J.; Kim, B.K.; Ricardez-Sandoval, L.; Yu, A. Pyrrolic-structure enriched nitrogen-doped graphene for highly efficient next-generation supercapacitors. J. Mater. Chem. A 2013, 1, 2904-2912. [CrossRef]

18. Biemolt, J.; Denekamp, I.M.; Slot, T.K.; Rothenberg, G.; Eisenberg, D. Boosting the supercapacitance of nitrogen-doped carbon by tuning surface functionalities. ChemSusChem 2017, 10, 4018-4024. [CrossRef]

19. Zhu, Y.; Chen, M.; Zhang, Y.; Zhao, W.; Wang, C. A biomass-derived nitrogen-doped porous carbon for high-energy supercapacitor. Carbon 2018, 140, 404-412. [CrossRef]

20. Li, H.F.; Wang, C.; Liu, L.F.; Xie, N.; Pan, M.; Wu, P.; Wang, X.D.; Zeng, Z.; Deng, S.; Dai, G.P. Facile one-step synthesis of N-doped carbon nanotubes/N-doped carbon nanofibers hierarchical composites by chemical vapor deposition. J. Nanoparticle Res. 2020, 22, 10. [CrossRef]

21. Sing, K.; Williams, R. Physisorption hysteresis loops and the characterization of nanoporous materials. Adsorp. Sci. Technol. 2004, 22, 773-782. [CrossRef]

22. Shahrokhian, S.; Mohammadi, R.; Asadian, E. One-step fabrication of electrochemically reduced graphene oxide/nickel oxide composite for binder-free supercapacitors. Int. J. Hydrog. Energy 2016, 41, 17496-17505. [CrossRef]

23. Frackowiak, E.; Lota, G.; Machnikowski, J.; Vix-Guterl, C.; Béguin, F. Optimisation of supercapacitors using carbons with controlled nanotexture and nitrogen content. Electrochim. Acta 2006, 51, 2209-2214. [CrossRef]

24. Kim, Y.-S.; Kumar, K.; Fisher, F.T.; Yang, E.-H. Out-of-plane growth of CNTs on graphene for supercapacitor applications. J. Nanotechnol. 2011, 23, 015301. [CrossRef]

25. Mombeshora, E.T.; Ndungu, P.G.; Jarvis, A.L.L.; Nyamori, V.O. Oxygen-modified multiwalled carbon nanotubes: Physicochemical properties and capacitor functionality. Int. J. Energy Res. 2017, 41, 1182-1201. [CrossRef]

26. Qian, X.; Li, N.; Imerhasan, M.; Wang, W. Conversion of low molecular weight hydrogel to nitrogen-doped carbon materials and its application as supercapacitor. Colloids Surf. A Physicochem. Eng. Asp. 2019, 573, 255-261. [CrossRef]

27. Mombeshora, E.T.; Ndungu, P.G.; Jarvis, A.L.L.; Nyamori, V.O. The physical and electrochemical properties of nitrogen-doped carbon nanotube- and reduced graphene oxide-titania nanocomposites. Mater. Chem. Phys. 2018, 213, 102-112. [CrossRef]

28. Han, X.; Jiang, H.; Zhou, Y.; Hong, W.; Zhou, Y.; Gao, P.; Ding, R.; Liu, E. A high performance nitrogen-doped porous activated carbon for supercapacitor derived from pueraria. J. Alloys Compd. 2018, 744, 544-551. [CrossRef]

29. Yang, M.; Zhou, Z. Recent breakthroughs in supercapacitors boosted by nitrogen-rich porous carbon materials. Adv. Sci. 2017, 4, 1600408. [CrossRef]

30. Ewels, C.; Glerup, M. Nitrogen doping in carbon nanotubes. J. Nanosci. Nanotechnol. 2005, 5, 1345-1363. [CrossRef]

31. Zhao, J.-R.; Jun, H.; Jiang-Feng, L.; Ping, C. N-doped carbon nanotubes derived from waste biomass and its electrochemical performance. Mater. Let. 2020, 261, 127146. [CrossRef]

32. Misnon, I.I.; Zain, N.K.M.; Aziz, R.A.; Vidyadharan, B.; Jose, R. Electrochemical properties of carbon from oil palm kernel shell for high performance supercapacitors. Electrochim. Acta 2015, 174, 78-86. [CrossRef] 
33. Zhang, G.; Chen, Y.; Chen, Y.; Guo, H. Activated biomass carbon made from bamboo as electrode material for supercapacitors. Mater. Res. Bull. 2018, 102, 391-398. [CrossRef]

34. Liou, T.-H.; Wu, S.-J. Characteristics of microporous/mesoporous carbons prepared from rice husk under base- and acid-treated conditions. J. Hazard. Mater 2009, 171, 693-703. [CrossRef] [PubMed]

35. Xu, X.; Wang, M.; Liu, Y.; Lu, T.; Pan, L. Metal-organic framework-engaged formation of a hierarchical hybrid with carbon nanotube inserted porous carbon polyhedra for highly efficient capacitive deionization. J. Mater. Chem. A 2016, 4, 5467-5473. [CrossRef] 